\title{
Subarachnoid Hemorrhage in Bacterial Meningitis Patients
}

\author{
Shahrzad S. Deliran Matthijs C. Brouwer Diederik van de Beek \\ Department of Neurology, Amsterdam UMC, University of Amsterdam, Amsterdam Neuroscience, Amsterdam, \\ The Netherlands
}

\section{Keywords}

Bacterial meningitis - Subarachnoid hemorrhage · Cerebrovascular complication · Stroke - Streptococcus pneumoniae

\begin{abstract}
Introduction: Subarachnoid hemorrhage (SAH) has been described as an uncommon complication of community-acquired bacterial meningitis. However, the incidence, clinical course, and outcome are unclear. Methods: We assessed the clinical characteristics, incidence, and clinical outcome of patients with $\mathrm{SAH}$ complicating bacterial meningitis in a prospective nationwide cohort study from 2006 to 2018 in the Netherlands. Patients were identified through the Netherlands Reference Laboratory for Bacterial Meningitis, which receives around $90 \%$ of CSF isolates of all Dutch patients with bacterial meningitis, or after direct report by the treating physician. Results: SAH was diagnosed in 22 of 2,306 episodes $(0.9 \%)$, of which $7(32 \%)$ were diagnosed upon admission and $15(68 \%)$ during admission. All patients showed clinical deterioration before SAH was diagnosed: altered mental status in 18 of 22 patients (82\%), focal neurological symptoms in $2(9 \%)$ and, new-onset fever with severe tachy-
\end{abstract}

cardia in 1 (5\%). Acute onset of headache was not reported in any of the patients. Distribution of blood was diffuse in the subarachnoid space in 7 patients (32\%), multifocal in 8 patients (36\%), and focal in 7 patients (32\%) of 22 patients. In 6 patients (27\%), CT angiography, MR angiography, or digital subtraction angiography was performed, showing a mycotic aneurysm in 1 patient (5\%) and vasculitis in 1 patient (5\%). Presence of SAH in bacterial meningitis patients was associated with a poor prognosis assessed at discharge: 12 of 22 patients with SAH died (54\%) compared to 361 of 2,257 $(16 \%, p<0.001)$ without $\mathrm{SAH}$, and 19 of 22 had an unfavorable outcome (86\%) compared to 831 of 2,257 (37\%, $p<$ 0.001). Conclusion: $\mathrm{SAH}$ is an uncommon complication in bacterial meningitis and is associated with high case fatality and morbidity.

(C) 2021 The Author(s)

Published by S. Karger AG, Basel

\section{Introduction}

Bacterial meningitis is a life-threatening disease with high mortality and morbidity $[1,2]$. Cerebrovascular complications are common in patients with bacterial meningitis and occur in up to $30 \%$, of which the majority karger@karger.com www.karger.com/ced

Karger $\stackrel{\text { ' }}{5}$

BOPEN ACCESS
(C) 2021 The Author(s)

Published by S. Karger AG, Basel

This is an Open Access article licensed under the Creative Commons Attribution-NonCommercial-4.0 International License (CC BY-NC) (http://www.karger.com/Services/OpenAccessLicense), applicable to the online version of the article only. Usage and distribution for commercial purposes requires written permission.
Correspondence to:

Diederik van de Beek, d.vandebeek@amsterdamumc.nl 
has cerebral infarctions, while intracerebral hemorrhages and cerebral sinus thrombosis are less frequent [3-5]. Subarachnoid hemorrhage (SAH) on cranial imaging has previously been reported in $20 \%$ of the hemorrhagic complications in bacterial meningitis [5]. Potential causes of the SAH consist of saccular [6] or mycotic aneurysms, but this is uncommonly reported as an underlying condition in bacterial meningitis patients [7-11]. In meningitis patients, other causes such as vasculitis may be considered as the cause of SAH [12-14]. However, it is unclear how often ancillary vascular imaging is performed (CT angiography, MR angiography, and digital subtraction angiography) to further study the cause of the SAH in these patients.

In patients without meningitis, $\mathrm{SAH}$ mostly occurs in patients with saccular aneurysms, which present with subacute headache, altered mental status, and/or neurologic deficits [6]. In bacterial meningitis patients, the clinical characteristics associated with SAH are unclear. SAH on cranial imaging may be a sign of severe vascular complications, as an autopsy study on 31 patients with pneumococcal meningitis showed parenchymal (micro) hemorrhages in $77 \%$ of the cases and signs of vascular inflammation in $94 \%$. However, there were no significant correlations between neuroimaging with the pathological findings [13]. We present the clinical characteristics, radiological findings, and outcome in SAH complicating bacterial meningitis in a nationwide cohort study on community-acquired bacterial meningitis in the Netherlands.

\section{Methods}

In a nationwide prospective cohort study on community-acquired bacterial meningitis in the Netherlands between March 2006 and July 2018, we identified patients older than 16 years with culture-proven bacterial meningitis or with CSF abnormalities suggestive for bacterial meningitis (CSF-blood glucose ratio $<0.23$, glucose level $<1.9 \mathrm{mmol} / \mathrm{L}$, CSF protein level $>2.2 \mathrm{~g} / \mathrm{L}$, leukocytes $>2,000 \times 10^{6} / \mathrm{L}$, polymorphonuclear leukocytes $>1,180 \times 10^{6} / \mathrm{L}$ ) [15]. The majority of these patients were listed in the Netherlands Reference Laboratory for Bacterial Meningitis (NRLBM), and some were reported by their physician. NRLBM receives around $90 \%$ of CSF isolates of all Dutch patients with bacterial meningitis. Daily updates from the hospitals where patients with bacterial meningitis were registered were presented to the investigators. Treating physicians were contacted by telephone and were requested to include their patients in this study. It was also possible for physicians to contact the investigators to include patients in the study. This study was approved by the Medical Ethics Committee of the Academic Medical Center in the Netherlands. In all cases, written informed consent was acquired from the patient or legal representative. Patients who underwent a neurosurgical operation, head trauma within 1 month prior to bacterial meningitis, and patients with neurosurgical device in situ or hospital-acquired meningitis were excluded. Patients were considered immunocompromised when there was a history of HIV infection, alcoholism, diabetes mellitus, splenectomy, or active immunosuppressive drug use. The clinical data were prospectively obtained by an online case record form (CRF). SAH was recorded in the cerebrovascular complication section of the CRF and was mentioned as an additional neuroimaging abnormality or complication separately.

Neuroimaging of patients registered to have SAH in the CRF was obtained and reevaluated by the investigators (S.S.D. and M.C.B.) to determine the presence and distribution (diffuse or (multi)focal) of the SAH. The Hounsfield unit (HU) of the suspected hemorrhagic lesion, which is correlated with the density of hematoma/hemorrhage [16-18], was also measured. All scans were additionally assessed on sinus and mastoid opacification, sulcal effacement, obliteration of cisterns, brain edema, cerebral infarction, cerebral venous thrombosis, intracerebral and intraventricular hemorrhage, brain abscess, empyema, hydrocephalus, (mycotic)aneurysm, and vasculitis.

At discharge, standard neurological examination was performed by the treating physician, and the functional outcome was scored according to the Glasgow Outcome Scale (GOS), a functional outcome scale with scores from 1 (death) to 5 (mild or no disability) [19]. A score of 1-4 is considered an unfavorable outcome and a score of 5 is considered a favorable outcome.

To describe the population characteristics, we used medians and interquartile ranges (IQR). Nonparametric testing was used to determine differences in continuous variables between the cases with or without SAH. The $\chi^{2}$ test and Fisher exact test were used to compare the categorical variables. Values of $p<0.05$ were considered to be statistically significant, and all statistical tests were 2 -tailed. The statistical analysis was conducted with IBM SPSS Statistics (version 25).

\section{Results}

SAH was registered in 24 of 2,306 episodes of bacterial meningitis as a complication. The patients were included after a report from the NRLBM in 2019 (87\%) episodes and 287 patients (12\%) were reported by their physician. In 22 patients $(0.9 \%$ [ $95 \%$ confidence interval: $0.6-1.4 \%]$ ), the neuroimaging was available for reevaluation and SAH could be confirmed. SAH was observed on admission in 7 of 22 patients (32\%), and the remaining 15 patients developed SAH during hospitalization (68\%) after a median of 5 days (IQR $1-7$ days). Ten patients (45\%) were female, and the median age was 65 (IQR 37-81) years. The presenting characteristics of patients with $\mathrm{SAH}$ and without SAH were comparable (Tables 1,2), except for seizures, which were more often present in patients with SAH $(28 \%$ vs. $7 \%, p=0.004)$. 
In $50 \%$ of $\mathrm{SAH}$ patients, there was a predisposing condition for bacterial meningitis present. ENT infections were reported in 8 of 22 patients (36\%), consisting of otitis media in $6(27 \%)$ and sinusitis in $3(14 \%)$. Endocarditis was reported in 1 patient (5\%). Three patients (14\%) used platelet aggregation inhibitors and 2 (9\%) anticoagulation upon admission (all vitamin $\mathrm{K}$ antagonists).

Altered level of consciousness defined as Glasgow Coma Scale (GCS) <14 was present in 14 of 22 patients $(77 \%)$, and 6 of 22 patients (27\%) presented in a coma defined as GCS $<8$. Focal neurological deficits were present in 11 of 22 patients (50\%). The triad of fever, neck stiffness, and altered level of consciousness was reported in 6 of 22 patients (27\%). Thrombocytopenia (defined as $\left.<150,000 / \mu \mathrm{L}\left[150 \times 10^{9} / \mathrm{L}\right]\right)$ was more frequent in the SAH group compared to patients without SAH (12 of 22 [55\%] vs. 525 of 2,140 [25\%]; $p<0.001$; Table 2).

Lumbar puncture was performed in all patients. The median white cell count in CSF was 1,568 cells $/ \mathrm{mm}^{3}$ (IQR 209-3,729), CSF-to-blood glucose ratio was 0.10 (IQR $0.09-0.45$ ), and the median protein level was 4.71 (IQR $3.02-9.01)$. CSF leukocyte count $<1,000$ cells $/ \mathrm{mm}^{3}$ was present in 8 of 22 patients (36\%). Cultures yielded Streptococcus pneumoniae as the causative pathogen in 13 of 22 patients (59\%), followed by Staphylococcus aureus, Haemophilus influenzae, and Listeria monocytogenes in 2 patients each (9\%), and Klebsiella pneumoniae, Streptococcus agalactiae, and Escherichia coli all in 1 patient (5\%).

The diagnosis of SAH was made by cranial CT in all patients. Three patients (14\%) received additional cerebral MRI. SAH was seen on the first scan in 9 patients (41\%), and the remaining cases were seen on the second scan during hospitalization. The repeated scans were made because of clinical deterioration in all patients, which consisted of an altered level of consciousness in 18 of 22 patients ( $82 \%)$, focal neurological deficits in $2(9 \%)$, and new-onset high-grade fever with severe tachycardia in 1 patient (5\%). In 1 patient, the nature of the clinical deterioration was not specified. Acute onset headache was not reported in any of the patients. Six patients (27\%) had additional CT angiography, showing a mycotic aneurysm of the medial cerebral artery in 1 patient $(5 \%)$ who also suffered from infectious endocarditis. Digital subtraction angiography was conducted in 1 patient, showing vascular irregularities consistent with vasculitis (5\%). SAH had a diffuse pattern on neuroimaging in 7 (32\%), multifocal in $8(36 \%)$, and focal in 7 of 22 patients $(32 \%$; shown in Fig. 1). Other neuroimaging findings included
Table 1. Characteristics of patients in SAH group $(n=22)$

\begin{tabular}{|c|c|}
\hline Patient characteristics & \\
\hline Age (IQR), years & $65(37-81)$ \\
\hline Female, $n(\%)$ & $10 / 22(45)$ \\
\hline Predisposing conditions for bacterial mening & tis, $n(\%)$ \\
\hline Sinusitis & $3 / 22(14)$ \\
\hline Otitis & $6 / 22(27)$ \\
\hline Endocarditis & $1 / 22(5)$ \\
\hline Immunocompromised state & $8 / 26(31)$ \\
\hline HIV positive & $1 / 22(5)$ \\
\hline Anticoagulant use prior admission & $2 / 22(9)$ \\
\hline $\begin{array}{l}\text { Platelet aggregation inhibitor use prior } \\
\text { admission }\end{array}$ & $3 / 22(14)$ \\
\hline Signs and symptoms on admission, $n(\%)$ & \\
\hline Headache & $11 / 22(50)$ \\
\hline Nausea & $5 / 22(23)$ \\
\hline Neck stiffness & $11 / 22(50)$ \\
\hline $\begin{array}{l}\text { Triad of neck stiffness, fever, and altered } \\
\text { mental status }\end{array}$ & $6 / 22(27)$ \\
\hline Temperature $\geq 38^{\circ} \mathrm{C}$ & $13 / 22(59)$ \\
\hline Seizures & $6 / 22(27)$ \\
\hline Altered mental status GCS $<14$ & $17 / 22(77)$ \\
\hline Coma GCS $<8$ & $6 / 22(27)$ \\
\hline Focal neurological deficit & $11 / 22(50)$ \\
\hline CSF parameters $^{\dagger}$ & \\
\hline Leucocyte count, cells $/ \mathrm{mm}^{3}$ & $1,568(209-3,729)$ \\
\hline Leucocyte count $<1,000$ cells $/ \mathrm{mm}^{3}, n(\%)$ & $8 / 22(36)$ \\
\hline Protein, g/L & $4.71(3.02-9.01)$ \\
\hline CSF-blood glucose ratio & $0.10(0.00-0.45)$ \\
\hline Cultures, $n(\%)$ & \\
\hline Streptococcus pneumoniae & $13 / 22(59)$ \\
\hline Staphylococcus aureus & $2 / 22(9)$ \\
\hline Haemophilus influenzae & $2 / 22(9)$ \\
\hline Listeria monocytogenes & $2 / 22(9)$ \\
\hline Other pathogens $\$^{\ddagger}$ & $3 / 22(14)$ \\
\hline Blood results $\$$ & \\
\hline Leucocyte count, cells $/ \mathrm{mm}^{3}$ & $16(12-25)$ \\
\hline C-reactive protein, $\mathrm{mg} / \mathrm{L}$ & $208(136-370)$ \\
\hline Thrombocyte count, platelets $/ \mathrm{mm}^{3}$ & $134(90-208)$ \\
\hline
\end{tabular}

$\mathrm{SAH}$, subarachnoid hemorrhage; IQR, interquartile range; GCS, Glasgow Coma Scale. ${ }^{\dagger}$ CSF leukocyte count and protein level were known for all 22 patients, and CSF-to-blood glucose ratio was known in 21 patients $(95 \%){ }^{\ddagger}$ Klebsiella pneumonia, Streptococcus agalactiae, and Escherichia coli all in 1 case. ${ }^{\S}$ Leucocyte count was known in all 22 patients, and c-reactive protein and thrombocyte count were known in 21 patients (95\%).

cerebral infarctions in 6 patients (27\%), cerebral edema in 7 patients (32\%), brain abscess in 1 patient (5\%), intraparenchymal hemorrhage in 2 patients (9\%), and hydrocephalus in 1 patient ( $5 \%$; Table 3 ). The median HU value measured in the suspected hemorrhagic lesion was 60 (45-66), consistent with blood [16, 18]. 
Table 2. Comparison of SAH to non-SAH group

\begin{tabular}{lccc}
\hline Characteristics & SAH $(n=22)$ & Non-SAH $(n=2,306)$ & $p$ value \\
\hline Age $(\mathrm{IQR})$, years & $65(37-81)$ & $61(48-70)$ & 0.4 \\
Female, $n(\%)$ & $10 / 22(45)$ & $1,109 / 2,284(48)$ & 0.7 \\
Endocarditis, $n(\%)$ & $1 / 22(5)$ & $32 / 2,164(1.2)$ & 0.3 \\
Thrombocytopenia $<150 \times 10^{9} / \mathrm{L}, n(\%)$ & $12 / 22(55)$ & $525 / 2,140(25)$ & $<0.001$ \\
GCS on admission & $10(8-13)$ & $11(9-14)$ & 0.7 \\
Prior anticoagulant use, $n(\%)$ & $5 / 22(23)$ & $319 / 2,284(14)$ & 0.2 \\
Complications during admission, $n(\%)$ & & & $<0.001$ \\
$\quad$ Seizures & $12 / 22(54)$ & $289 / 2,154(13)$ & $<0.001$ \\
$\quad$ Impaired consciousness & $20 / 22(95)$ & $1,085 / 2,074(52)$ & $<0.001$ \\
$\quad$ Circulatory shock & $8 / 22(36)$ & $208 / 2,131(10)$ & $<0.001$ \\
$\quad$ Respiratory failure & $12 / 22(55)$ & $531 / 2,180(24)$ & $<0.001$ \\
$\quad$ Transfer to ICU & $19 / 22(91)$ & $861 / 2,168(40)$ & $<0.001$ \\
$\quad$ Focal neurological deficit & $11 / 18(61)$ & $464 / 2,077(22)$ & $<0.001$ \\
Clinical Outcome, $n(\%)$ & & & $<0.001$ \\
$\quad$ Death & $12 / 22(54)$ & $361 / 2,257(16)$ & $831 / 2,257(37)$ \\
$\quad$ Unfavorable outcome & $19 / 22(86)$ & & \\
\hline
\end{tabular}

SAH, subarachnoid hemorrhage; IQR, interquartile range; GCS, Glasgow Coma Scale.

Fig. 1. Neuroimaging axial CT showing SAH in a diffuse pattern (a); axial CT showing multifocal hemorrhagic lesions in the subarachnoid space (arrows) (b); axial CT showing focal lesion in the subarachnoid space in the left parietal region (arrow) (c); CT angiography maximum intensity projection showing a mycotic aneurysm of the right medial cerebral artery (arrow) (d). SAH, subarachnoid hemorrhage.

Subarachnoid Hemorrhage in Meningitis

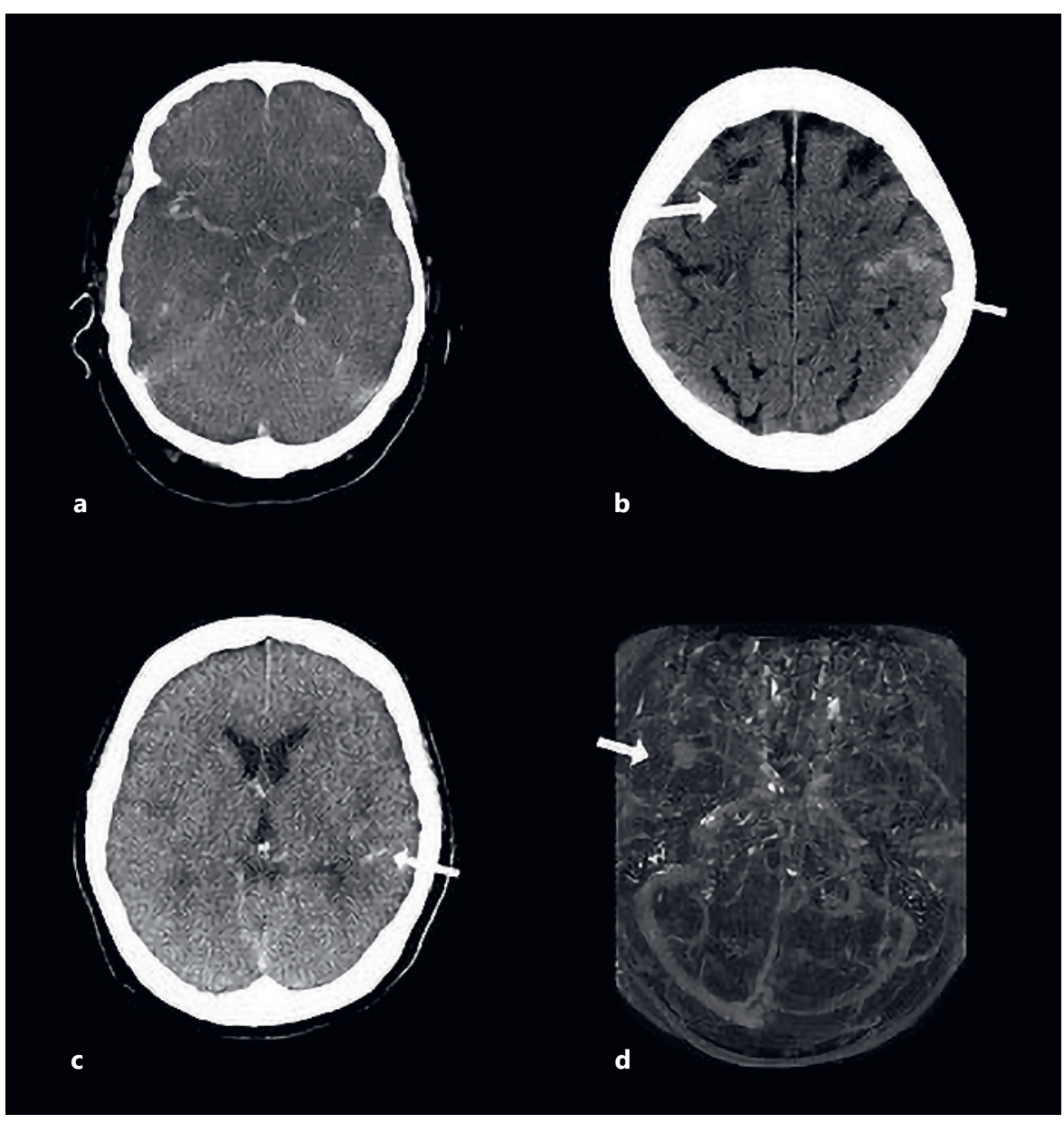

Cerebrovasc Dis 2022;51:118-124 
All patients received empirical antimicrobial therapy; 20 of 22 patients (90\%) received a third-generation cephalosporin often combined with amoxicillin (15 of 22 patients, 68\%). The remaining 2 patients (9\%) received penicillin monotherapy. Adjunctive dexamethasone therapy was administered in 18 of 22 patients (82\%), of whom 14 patients received $10 \mathrm{mg} 6$ hourly for 4 days. One patient underwent a decompressive craniectomy because of multiple brain abscesses and cerebral empyema (5\%), 3 of 22 patients (14\%) had a mastoidectomy, and 1 patient underwent a tympanocentesis (5\%). One patient was treated for hydrocephalus with an external ventricular drain.

Most of the SAH patients developed complications during admission (Table 3), which consisted of newly impaired level of consciousness in 20 of 22 patients (90\%), respiratory failure in 12 of $22(54 \%)$, and circulatory shock in 8 of $22(36 \%)$. Twelve patients of $22(54 \%)$ developed seizures during admission and 11 developed focal neurological deficits (54\%). Twelve of $22 \mathrm{SAH}$ patients died (54\%) compared to 361 of 2,257 (16\%) in the nonSAH group $(p<0.001)$. The functional outcome defined by GOS assessed at discharge was unfavorable in the $\mathrm{SAH}$ group, with a score of $1-4$ in 19 of 22 patients (86\%) compared to 831 of $2,257(37 \%)$ in non-SAH patients $(p<$ $0.001)$. Six of $10 \mathrm{SAH}$ survivors $(60 \%)$ had neurological deficits at discharge, consisting of cognitive impairment in 5 , aphasia in 2, paresis in 2, and sensory changes in 1 , and present Babinski reflex in 3.

\section{Discussion}

This study shows that $\mathrm{SAH}$ is a rare but severe complication of community-acquired bacterial meningitis with high mortality (54\%) and unfavorable outcome (86\%), occurring in $0.9 \%$ of cases. Previous studies described the presence of intracerebral hemorrhage in bacterial meningitis in 2-9\% cases in a tertiary referral center but did not specify whether these included SAH patients $[3,20]$. A study in adults with pneumococcal meningitis described SAH in 4 of 87 patients (5\%) [14]. The higher rate in this study can be explained as their patients were admitted to an ICU being more severely affected. Furthermore, the study was performed before dexamethasone was introduced as standard treatment, which reduces neurological complications in bacterial meningitis [21].

All patients with SAH showed clinical deterioration before SAH being diagnosed, both on admission and during hospitalization. This deterioration was not accompanied by acute headache, which is the predominant symp-
Table 3. Neuroimaging findings, clinical outcome, and neurological sequelae of SAH group $(n=22)$

\begin{tabular}{lc}
\hline SAH group & \\
\hline Neuroimaging findings, $n(\%)$ & \\
Recent cerebral infarction & $6 / 22(27)$ \\
Edema & $7 / 22(32)$ \\
Brain abscess & $1 / 22(5)$ \\
Suggestive for pseudo-SAH & $9 / 22(41)$ \\
Subdural effusion & $3 / 22(14)$ \\
Mycotic aneurysm & $1 / 22(5)$ \\
Hydrocephalus & $1 / 22(5)$ \\
(Multi)focal signs of SAH & $15 / 22(68)$ \\
Diffuse signs of SAH & $7 / 22(32)$ \\
HU value for SAH & $60(45-66)$ \\
Clinical outcome, Glasgow Outcome Scale score, $n(\%)$ \\
Death & $12 / 22(55)$ \\
Persistent vegetative state & $0 / 22$ \\
Severe disability & $4 / 22(18)$ \\
Moderate disability & $3 / 22(14)$ \\
Low disability & $3 / 22(14)$ \\
Neurological sequelae at discharge $(n=10)$ & \\
Cognitive impairment & $5 / 10(50)$ \\
Aphasia & $2 / 10(20)$ \\
Ataxia & $1 / 10(10)$ \\
Sensory deficit & $1 / 10(10)$ \\
Cranial nerve palsy & $1 / 10(10)$ \\
Paresis & $2 / 10(20)$ \\
Hearing impairment & $4 / 10(40)$ \\
Neurologic deficit on discharge & $6 / 10(60)$ \\
& \\
\hline
\end{tabular}

SAH, subarachnoid hemorrhage; HU, Hounsfield unit.

tom of aneurysmal SAHs [6]. This is consistent with the different pathogenesis of SAH in bacterial meningitis patients, which is presumably due to vascular inflammation instead of cerebral aneurysms. The lack of acute headache during deterioration implies that $\mathrm{SAH}$ will often not be suspected prior to the imaging, and it may be considered a coincidental finding. Repeated neuroimaging in bacterial meningitis patients showing clinical deterioration should be conducted to determine the underlying cause [22] such as SAH. Ideally, imaging of the blood vessels with MR angiography or digital subtraction angiography should be performed to identify vascular abnormalities that may have treatment implications.

A neuropathology study suggested an increase in the number of infectious intracranial mycotic aneurysms in patients with bacterial meningitis [23]. Mycotic intracranial aneurysms are $0.7-5.4 \%$ of all intracranial aneurysms [24], and meningitis is thought to be the underlying cause in 5\% of them [25]. The majority of mycotic intracranial aneurysms develop in patients with bacterial endocarditis 
(65\%) [25]. A study performing digital subtraction angiography in patients with bacterial meningitis and SAH showed vascular wall irregularities matching with intracerebral vasculitis [14]. Inflammatory mechanisms such as intracerebral vasculitis and mycotic aneurysms are probably causing $\mathrm{SAH}$ in bacterial meningitis. Individualized decisions regarding medical versus surgical treatment are recommended in mycotic aneurysms complicating bacterial meningitis, considering factors such as age, location, the number of the aneurysm(s), the condition of the patient, and predisposing factors [7]. Administering corticosteroids to diminish the inflammatory response in intracerebral infectious vasculitis has been suggested earlier, although this should be decided on a case-to-case basis, considering the lack of randomized controlled trials [26-28].

This study has several limitations. First, the study was observational, and routine neuroimaging was not performed in all patients; therefore, the time of neuroimaging and modality used were variables (CT, CT with contrast, MRI, and DSA). The lack of uniformity neuroimaging in SAH patients could underestimate the patients with vasculitis or mycotic aneurysms. Further, cranial imaging was not conducted in all 2,306 patients in the cohort and could have led to an underestimation of the SAH cases. Another limitation of our study is that we were not able to obtain the neuroimaging to be re-evaluated 2 patients and the patients were excluded from the study. We conclude that $\mathrm{SAH}$ is an uncommon but severe complication in bacterial meningitis, which presents with clinical deterioration without an acute headache and is associated with high case fatality and morbidity.

\section{Acknowledgements}

We are thankful to all the Dutch physicians and patients who participated in the MeninGene study.

\section{Statement of Ethics}

This study has been approved by the Medical Ethics Committee of the Academic Medical Center in the Netherlands (METC201343) and has therefore been performed in accordance with the ethical standards laid down in the 1964 Declaration of Helsinki and its later amendments. All subjects or their legal representatives gave informed consent prior to their inclusion in this study.

\section{Conflict of Interest Statement}

The authors declare that they have no conflicts of interest.

\section{Funding Sources}

This study was supported by the Netherlands Organization for Health Research and Development (ZonMw; NWO-Vici-Grant [91819627] to D.B. and NWO-Vidi-Grant [916.13.078] to M.C.B.) and the European Research Council (European Research Council Starting Grant to D.B.). The Netherlands Reference Laboratory is funded by the National Institute of Public Health and the Environment.

\section{Author Contributions}

S.S.D made substantial contributions to the conception of the work, analysis and interpretation of data, drafted the work, approved the version to be published, and agrees to be accountable for all aspects of the work. M.C.B. and D.B. made substantial contributions to the design of the work, interpretation of data, revised it critically for important intellectual content, approved the version to be published, and agree to be accountable for all aspects of the work.

\section{Data Availability Statement}

The anonymized data are available on request via www.MeninGene.eu.

\section{References}

1 Bijlsma MW, Brouwer MC, Kasanmoentalib ES, Kloek AT, Lucas MJ, Tanck MW, et al. Community-acquired bacterial meningitis in adults in the Netherlands, 2006-14: a prospective cohort study. Lancet Infect Dis. 2016;16(3): 339-47.

2 van de Beek D, Brouwer M, Hasbun R, Koedel U, Whitney CG, Wijdicks E. Community-acquired bacterial meningitis. Nat Rev Dis Primers. 2016;2:16074.

3 Pfister HW, Borasio GD, Dirnagl U, Bauer M, Einhäupl KM. Cerebrovascular complications of bacterial meningitis in adults. Neurology. 1992;42(8):1497-504.
4 Weisfelt M, van de Beek D, Spanjaard L, Reitsma JB, de Gans J. Clinical features, complications, and outcome in adults with pneumococcal meningitis: a prospective case series. Lancet Neurol. 2006;5(2):1239.

5 Mook-Kanamori BB, Fritz D, Brouwer MC, van der Ende A, van de Beek D. Intracerebral hemorrhages in adults with community associated bacterial meningitis in adults: should we reconsider anticoagulant therapy? PLoS One. 2012;7(9):e45271.

6 Muehlschlegel S. Subarachnoid hemorrhage. Continuum. 2018;24(6):1623-57.
7 Kannoth S, Iyer R, Thomas SV, Furtado SV, Rajesh BJ, Kesavadas C, et al. Intracranial infectious aneurysm: presentation, management and outcome. J Neurol Sci. 2007;256(12):3-9.

8 Minnerup J, Schilling $M$, Wersching $\mathrm{H}$, Olschläger C, Schäbitz WR, Niederstadt T, et al. Development of a mycotic aneurysm within 4 days. Neurology. 2008;71(21):1745.

9 Chun JY, Smith W, Halbach VV, Higashida RT, Wilson CB, Lawton MT. Current multimodality management of infectious intracranial aneurysms. Neurosurgery. 2001;48(6): $1203-4$. 
10 Clare CE, Barrow DL. Infectious intracranial aneurysms. Neurosurg Clin N Am. 1992;3(3): 551-66.

11 van Gijn J, Kerr RS, Rinkel GJ. Subarachnoid haemorrhage. Lancet. 2007;369(9558):30618.

12 Koda Y, Seto Y, Takeichi S, Kimura H. Fatal subarachnoid hemorrhage complicating actinomycotic meningitis. Forensic Sci Int. 2003; 134(2-3):169-71.

13 Engelen-Lee JY, Brouwer MC, Aronica E, van de Beek D. Pneumococcal meningitis: clinical-pathological correlations (MeninGenePath). Acta Neuropathol Commun. 2016;4: 26.

14 Kastenbauer S, Pfister HW. Pneumococcal meningitis in adults: spectrum of complications and prognostic factors in a series of 87 cases. Brain. 2003;126(Pt 5):1015-25.

15 Spanos A, Harrell FE Jr, Durack DT. Differential diagnosis of acute meningitis. An analysis of the predictive value of initial observations. JAMA. 1989;262(19):2700-7.

16 Scott WR, New PF, Davis KR, Schnur JA. Computerized axial tomography of intracerebral and intraventricular hemorrhage. Radiology. 1974;112(1):73-80.
17 Chakeres DW, Bryan RN. Acute subarachnoid hemorrhage: in vitro comparison of magnetic resonance and computed tomography. AJNR Am J Neuroradiol. 1986 Mar;7(2): 223-8.

18 Norman D, Price D, Boyd D, Fishman R, Newton TH. Quantitative aspects of computed tomography of the blood and cerebrospinal fluid. Radiology. 1977;123(2):335-8.

19 Jennett B, Teasdale G, Braakman R, Minderhoud J, Knill-Jones R. Predicting outcome in individual patients after severe head injury. Lancet. 1976;1(7968):1031-4.

20 Pfister HW, Feiden W, Einhäupl KM. Spectrum of complications during bacterial meningitis in adults. Results of a prospective clinical study. Arch Neurol. 1993;50(6):575-81.

21 Brouwer MC, McIntyre P, Prasad K, van de Beek D. Corticosteroids for acute bacterial meningitis. Cochrane Database Syst Rev. 2015(9):CD004405.

22 van de Beek D, Cabellos C, Dzupova O, Esposito S, Klein M, Kloek AT, et al. ESCMID guideline: diagnosis and treatment of acute bacterial meningitis. Clin Microbiol Infect. 2016;22(Suppl 3):S37-62.
23 Engelen-Lee JY, Brouwer MC, Aronica E, van de Beek D. Delayed cerebral thrombosis complicating pneumococcal meningitis: an autopsy study. Ann Intensive Care. 2018;8(1): 20.

24 Nakahara I, Taha MM, Higashi T, Iwamuro Y, Iwaasa M, Watanabe Y, et al. Different modalities of treatment of intracranial mycotic aneurysms: report of 4 cases. Surg Neurol. 2006;66(4):405-10

25 Ducruet AF, Hickman ZL, Zacharia BE, Narula R, Grobelny BT, Gorski J, et al. Intracranial infectious aneurysms: a comprehensive review. Neurosurg Rev. 2010;33(1):37-46.

26 Pugin D, Copin JC, Goodyear MC, Landis T, Gasche Y. Persisting vasculitis after pneumococcal meningitis. Neurocrit Care. 2006;4(3): $237-40$.

27 Vergouwen MD, Schut ES, Troost D, van de Beek D. Diffuse cerebral intravascular coagulation and cerebral infarction in pneumococcal meningitis. Neurocrit Care. 2010;13(2): $217-27$

28 Lucas MJ, Brouwer MC, van de Beek D. Delayed cerebral thrombosis in bacterial meningitis: a prospective cohort study. Intensive Care Med. 2013;39(5):866-71. 